International Journal of Molecular Sciences

ISSN 1422-0067

Article

www.mdpi.com/journal/ijms

\title{
Explaining Ionic Liquid Water Solubility in Terms of Cation and Anion Hydrophobicity
}

\author{
Johannes Ranke $^{1, \star}$, Alaa Othman ${ }^{2}$, Ping Fan $^{1}$ and Anja Müller ${ }^{3}$ \\ ${ }^{1}$ Abteilung Nachhaltigkeit in der Chemie, UFT Zentrum für Umweltforschung und \\ Umwelttechnologie, Universität Bremen, Postfach 330440, 28334 Bremen, Germany; E-Mail: \\ pingfan@web.de \\ ${ }^{2}$ Medical biochemistry department, Kasr Alainy faculty of medicine Cairo University, Cairo, Egypt; \\ E-Mail: alaaothman@hotmail.com \\ ${ }^{3}$ School of Engineering and Science, Jacobs University Bremen, Postfach 750561, 28725 Bremen, \\ Germany; E-Mail: anj.mueller@jacobs-university.de
}

* Author to whom correspondence should be addressed; E-Mail: jranke@uni-bremen.de

Received: 12 February 2009; in revised form: 11 March 2009 / Accepted: 16 March 2009 /

Published: 18 March 2009

\begin{abstract}
The water solubility of salts is ordinarily dictated by lattice energy and ion solvation. However, in the case of low melting salts also known as ionic liquids, lattice energy is immaterial and differences in hydrophobicity largely account for differences in their water solubility. In this contribution, the activity coefficients of ionic liquids in water are split into cation and anion contributions by regression against cation hydrophobicity parameters that are experimentally determined by reversed phase liquid chromatography. In this way, anion hydrophobicity parameters are derived, as well as an equation to estimate water solubilities for cation-anion combinations for which the water solubility has not been measured. Thus, a new pathway to the quantification of aqueous ion solvation is shown, making use of the relative weakness of interactions between ionic liquid ions as compared to their hydrophobicities.
\end{abstract}

Keywords: Ionic liquids; Water solubility; Cations; Anions; Lipophilicity. 


\section{Introduction}

The presumed discovery of the first ionic liquid by Walden [1] took place in 1914. After that, it took over 80 years for the bloom of ionic liquid research to appear, starting with the discovery of a new class of air and water stable ionic liquids by Wilkes in 1992 [2]. It will maybe take another 80 years to realize the full potential of these substances, or, as Uwe Vagt (BASF) put it at the Intertech Pira Conference on Ionic Liquids in Prague in October 2007, "We are still at the beginning." Numerous contributions [3, 4, 5, 6, 7, 8] have increased our understanding of basic properties, and to predict physical $[9,10,11,12]$ or toxicological $[13,14,15]$ properties of ionic liquids. Especially in the context of green and sustainable chemistry, there is a vision of a molecular design of ionic liquids which would combine technical advantages with a set of favorable properties like ready availability and low risk for health and environment $[16,17]$.

The quality of our recently found correlation between cation hydrophobicity and cytotoxicity for ionic liquids with relatively small anions [14] inspired us to also investigate the correlation of cation hydrophobicity with water solubility data at $293 \mathrm{~K}$ that had previously been generated in our labs. Because of the good correlation found within this dataset (see below), we complemented our water solubility data with data from the scientific literature.

The model we use for the water solubility of ionic liquids is:

$$
\log x_{\mathrm{IL}, \mathrm{w}}^{\mathrm{sat}}=-m \log k_{0, \mathrm{c}}+c_{\mathrm{a}}+\epsilon,
$$

where $x_{\mathrm{IL}, \mathrm{w}}^{\mathrm{sat}}$ is the mole fraction solubility of the ionic liquid at $293 \mathrm{~K}, k_{0, \mathrm{c}}$ is the cation hydrophobicity parameter as defined previously [14], $c_{\mathrm{a}}$ is an anion specific constant expressing their hydrophobicity, and $\epsilon$ is the random variable describing deviations from the model with unknown or unspecified causes. The slope $m$ should ideally be unity if all model assumptions are satisfied.

Briefly, the model is based on the idea that the excess molar free energy of dissolving the ionic liquid in water $\Delta_{\mathrm{IL}, \mathrm{w}} g^{\mathrm{E}}=R T \ln \gamma_{\mathrm{IL}, \mathrm{w}}$ can be expressed as the sum of a cation and an anion contribution, where the contribution of the cation is given by $\log k_{0, \mathrm{c}}$ plus an unknown constant, and the contribution of the anion is extracted by a least squares fit of the model to the data.

\section{Theory}

For the theoretical discussion of the model we need a notation that covers both a) cation partitioning in reversed phase HPLC, quantified by the capacity factor $k_{\mathrm{c}}$ extrapolated to the conditions at the beginning of an acetonitrile gradient and b) the ionic liquid - water equilibrium at saturation, specified by the equilibrium mole fraction of ionic liquid in the water phase $x_{\mathrm{w}}^{\mathrm{sat}}$.

We first denote the chemical potential of an ionic liquid in solvent $s$ as:

$$
\mu_{\mathrm{IL}, \mathrm{s}}=\mu_{\mathrm{IL}}^{\bullet}+R T \ln \gamma_{\mathrm{IL}, \mathrm{s}} x_{\mathrm{IL}, \mathrm{s}}
$$

using the pure ionic liquid as the standard state. Restricting our model to ionic liquids that are composed of one cation type and one anion type at a 1:1 ratio (covering the majority of what is called ionic liquids today), we split $\mu_{\mathrm{IL}, \mathrm{s}}$ into the sum of the chemical potentials of cation and anion: 


$$
\mu_{\mathrm{IL}, \mathrm{s}}=\mu_{\mathrm{c}, \mathrm{s}}+\mu_{\mathrm{a}, \mathrm{s}}
$$

with

$$
\begin{aligned}
& \mu_{\mathrm{c}, \mathrm{s}}=\mu_{\mathrm{c}}^{\bullet}+R T \ln \gamma_{\mathrm{c}, \mathrm{s}} x_{\mathrm{c}, \mathrm{s}} \\
& \mu_{\mathrm{a}, \mathrm{s}}=\mu_{\mathrm{a}}^{\bullet}+R T \ln \gamma_{\mathrm{a}, \mathrm{s}} x_{\mathrm{a}, \mathrm{s}}
\end{aligned}
$$

using the pure ionic liquid as the standard state as in equation 2. Note that these ion chemical potentials are not defined sensu strictu, because ions of one polarity can not be solvated without the presence of suitable counteranions. However, for our discussion it is convenient to use the terminology of chemical potentials and activity coefficients of ions, although extrathermodynamic assumptions are necessary to determine them [18].

We will now use this notation in order to discuss the two scenarios mentioned above, which are depicted in Figure 1:

Figure 1. Equilibria of ionic liquid and water (left) and of ionic liquid cations between stationary and mobile phase in buffered reversed phase HPLC (right). Open circles are ionic liquid cations and anions, grey circles are buffer ions present in the HPLC mobile phase. In the case of the water saturation, electroneutrality in the water can only be kept if the concentrations of IL cations and anions are equal. In the case of buffered HPLC, IL cations and anions can partition largely independent of each other if sufficient concentrations of counterions are present in both phases.
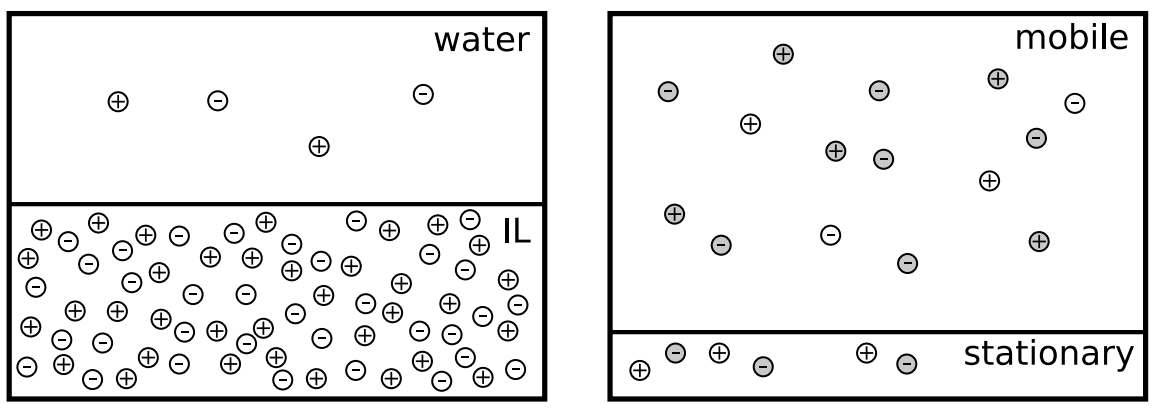

In the case of the saturation of water with an ionic liquid, electroneutrality dictates that $x_{\mathrm{c}, \mathrm{w}}=x_{\mathrm{a}, \mathrm{w}}=$ $x_{\mathrm{IL}, \mathrm{w}}$ in the aqueous phase $w$. Neglecting the amount of water partitioning into the $\mathrm{IL}\left(x_{\mathrm{IL}, \mathrm{IL}}^{\mathrm{sat}} \approx 1\right.$, Assumption 1), and assuming that it does not significantly influence the chemical environment of the IL $\left(\gamma_{\mathrm{IL}, \mathrm{IL}}^{\mathrm{sat}} \approx 1\right.$, Assumption 2$)$, the chemical potential of the IL at water saturation is approximately equal to the standard chemical potential of the standard state, i.e. $\mu_{\mathrm{IL}, \mathrm{IL}}^{\mathrm{sat}} \approx \mu_{\mathrm{IL}}^{\bullet}$. 
At the point of the saturation equilibrium, the chemical potentials of the IL have to be equal, i.e.

$$
\mu_{\mathrm{IL}, \mathrm{IL}}^{\mathrm{sat}}=\mu_{\mathrm{IL}, \mathrm{w}}^{\mathrm{sat}}
$$

Using the standard state as an approximation for the left hand side of equation 6, and equation 2 for its right hand side,

$$
\mu_{\mathrm{IL}}^{\bullet}=\mu_{\mathrm{IL}}^{\bullet}+R T \ln \gamma_{\mathrm{IL}, \mathrm{s}} x_{\mathrm{IL}, \mathrm{s}}
$$

we can rearrange the result to read

$$
R T \ln x_{\mathrm{IL}, \mathrm{w}}^{\mathrm{sat}}=-R T \ln \gamma_{\mathrm{IL}, \mathrm{w}}
$$

We now assume that the excess molar free energy of dissolving the IL in water $\Delta_{\mathrm{IL}, \mathrm{w}} g^{\mathrm{E}}=R T \ln \gamma_{\mathrm{IL}, \mathrm{w}}$ can be expressed as the sum of a cation and an anion contribution (Assumption 3), i.e.

$$
R T \ln x_{\mathrm{IL}, \mathrm{w}}^{\mathrm{sat}}=-R T \ln \gamma_{\mathrm{c}, \mathrm{w}}-R T \ln \gamma_{\mathrm{a}, \mathrm{w}}
$$

or, if we divide by $R T$ and move to base ten for the logarithmic expressions

$$
\log x_{\mathrm{IL}, \mathrm{w}}^{\mathrm{sat}}=-\log \gamma_{\mathrm{c}, \mathrm{w}}-\log \gamma_{\mathrm{a}, \mathrm{w}}
$$

which gives us a relation between cation and ion hydrophobicities, expressed by their activity coefficients in water, and water solubility.

If we look at the cation partitioning between mobile and stationary phases in reversed phase HPLC, we can assume that electroneutrality in both phases is guaranteed by a sufficient concentration of counteranions present in them (Assumption 4). This only appears reasonable if the buffer anion is sufficiently hydrophobic to be able to enter the stationary interphase at the chemically modified silica surface, or if there is a sufficient surface concentration of negatively charged sites with loosely bound counterions, for example dissociated silanol groups.

If this condition is satisfied, and if we neglect ion pairing between ionic liquid cations and anions (Assumption 5), we can denote the cation partitioning coefficient between stationary and mobile phase

$$
K_{\mathrm{c}, \mathrm{stm}}=\frac{x_{\mathrm{c}, \mathrm{st}}}{x_{\mathrm{c}, \mathrm{m}}}=\frac{\gamma_{\mathrm{c}, \mathrm{m}}}{\gamma_{\mathrm{c}, \mathrm{st}}}
$$

neglecting any electrostatic contribution to the partitioning. In logarithmic form, this can be written as

$$
\log K_{\mathrm{c}, \mathrm{stm}}=\log \gamma_{\mathrm{c}, \mathrm{m}}+c_{1}
$$

where the $c_{1}$ represents the contribution of the activity coefficient of the cation in the stationary phase, but can potentially also represent an electrostatic contribution, which would be independent of the type of the cation. If we assume that the activity coefficient of the cation in the stationary phase is approximately invariant for the range of cations studied (Assumption 6), $c_{1}$ can be taken as a constant.

In a previous study, we have shown how a measure for such a partition coefficient $K_{\mathrm{c}, \mathrm{stm}}$ can be extracted from retention times in gradient HPLC. More specifically, we use the capacity factor $\log k_{0, c}$ 
for the situation at the beginning of the gradient, i.e. for the situation where the concentration of organic modifier is zero, and the mobile phase only consists of an ammonium acetate buffer, as a measure for the cation partitioning coefficient $K_{\mathrm{c}, \mathrm{stm}_{0}}$, also at the beginning of the HPLC gradient [14].

In partition chromatography, the constant of proportionality $c_{2}$ in the equation for the capacity factor $k$

$$
k=c_{2} K_{\text {stm }}
$$

is just the phase ratio of stationary and mobile phase in the separation column. Since the volumes of stationary and mobile phase in reversed phase HPLC are ill-defined, we treat $c_{2}$ as a constant without explicit physical interpretation.

Combining equations 12 and 13 , we find that the $\log$ of the capacity factor $\log k_{0, \mathrm{c}}$ is just the cation activity coefficient in the mobile phase $m_{0}$ at the beginning of the gradient plus a constant

$$
\log k_{0, \mathrm{c}}=\log \gamma_{\mathrm{c}, \mathrm{m}_{0}}+c_{3} .
$$

The constant $c_{3}$ incorporates chemical interactions of the cations with the stationary phase, possibly an electrostatic contribution, and the correlate to the phase ratio $c_{2}$ from equation 13.

Under the final assumption that the activity coefficient of the cation in pure water is approximately equal to its activity coefficient in the mobile phase at the beginning of the gradient, in our case an ammonium acetate buffer, (Assumption 7), we can combine equations 10 and 14 to

$$
\log x_{\mathrm{IL}, \mathrm{w}}^{\mathrm{sat}}=-\log k_{0, \mathrm{c}}-\log \gamma_{\mathrm{a}, \mathrm{w}}+c_{3}
$$

Therefore the slope $m$ in the model equation 1 for the water solubility of ionic liquids repeated below is ideally equal to unity. The anion constant $c_{\mathrm{a}}$ equals $c_{3}-\log \gamma_{a, w}$.

$$
\log x_{\mathrm{IL}, \mathrm{w}}^{\mathrm{sat}}=-m \log k_{0, \mathrm{c}}+c_{\mathrm{a}}+\epsilon
$$

\section{Materials and Methods}

Ionic liquid nomenclature. In this study, the acronym scheme established at the UFT Center for Environmental Research and Sustainable Technology, University of Bremen is used for cations as illustrated in Table 1. Generally, numbers refer to alkyl chains (as in IM14 where the numbers refer to one methyl and one alkyl substituent on the nitrogens of the imidazolium cation) similar to their use in Wiswesser line notation. However, sometimes they are also pragmatically used to designate the site of substitution, as in Py4-3Me, where "-3Me" indicates 3-methyl substitution of the N-butylpyridinium cation. 
Table 1. Chemical structures of the cations treated in this study, along with their acronyms. In the acronyms, IM, Mor, N, Pip, Pyr and Py are used for the imidazolium, morpholinium, ammonium, piperidinium, pyrrolidinium and pyridinium head groups. $n$ designates linear alkyl chains with varying length, where $n$ is the number of carbon atoms in the chain. In the graphs, these chains correspond to the residues $\mathrm{R}$.

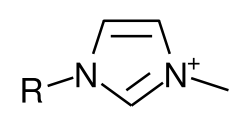

$\operatorname{IM} 1 n$

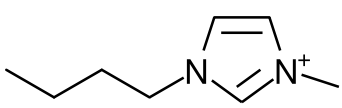

IM14<smiles>C[n+]1ccn(CCO)c1</smiles>

$\mathrm{IM} 12 \mathrm{OH}$

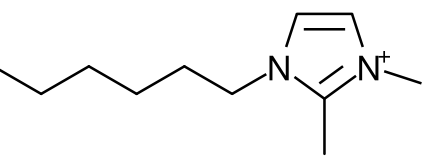

IM16-2Me<smiles>CCOC[N+]1(C)CCOCC1</smiles>

Mor11O2

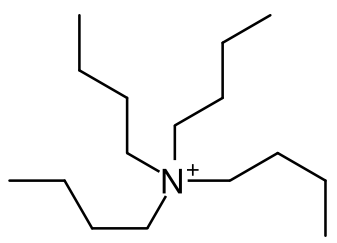

N4444

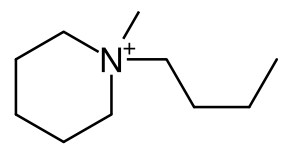

Pip14

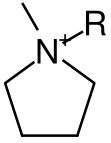

$\operatorname{Pyr} 1 n$<smiles>OCCC[n+]1ccccc1</smiles>

$\mathrm{Py} 3 \mathrm{OH}$

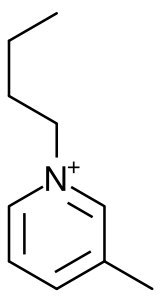

Py4-3Me<smiles>[R][n+]1ccc(C)cc1</smiles>

Pyn-4Me<smiles>CCCCCC[n+]1ccc(N(C)C)cc1</smiles>

Py6-4NMe2

Ionic liquids listed in Table 2 were used as received from Merck KGaA, Darmstadt, Germany. The first three entries in the table were used for the determination of their cation hydrophobicities as specified below. The remaining ionic liquids were used in the determination of their water solubility. 
Table 2. Ionic liquids used for generation of original data for this study.

\begin{tabular}{|c|c|}
\hline Ionic liquid & Acronym \\
\hline 1-ethyl-3-methylimidazolium chloride & $\mathrm{IM} 12 \mathrm{Cl}$ \\
\hline 1-(3-hydroxypropyl)pyridinium chloride & $\mathrm{Py} 3 \mathrm{OH} \mathrm{Cl}$ \\
\hline 4-(ethoxymethyl)-4-methylmorpholinium chloride & Mor11O2 Cl \\
\hline 1-butyl-3-methylimidazolium hexafluorophosphate & IM14 PF6 \\
\hline 1-butyl-3-methylimidazolium bis(trifluoromethylsulfonyl)imide & $\mathrm{IM} 14(\mathrm{CF} 3 \mathrm{SO} 2) 2 \mathrm{~N}$ \\
\hline 1-hexyl-3-methylimidazolium hexafluorophosphate & IM16 PF6 \\
\hline 1-hexyl-3-methylimidazolium bis(trifluoromethylsulfonyl)imide & IM16 (CF3SO2)2N \\
\hline 1-hexyl-3-methylimidazolium tris(trifluoromethylsulfonyl)methide & IM16 (CF3SO2)3C \\
\hline 1-hexyl-3-methylimidazolium trifluorotris(pentafluoroethyl)phosphate & IM16 (C2F5)3PF3 \\
\hline 1-octyl-3-methylimidazolium hexafluorophosphate & IM18 PF6 \\
\hline 1-octyl-3-methylimidazolium bis(trifluoromethylsulfonyl)imide & IM18 (CF3SO2)2N \\
\hline 1-(3-hydroxypropyl)pyridinium bis(trifluoromethylsulfonyl)imide & Рy3OH (CF3SO2)2N \\
\hline 4-(dimethylamino)-1-hexylpyridinium bis(trifluoromethylsulfonyl)imide & Py6-4NMe2 (CF3SO2)2N \\
\hline 1-butyl-1-methylpyrrolidinium bis(trifluoromethylsulfonyl)imide & Pyr14 (CF3SO2)2N \\
\hline 1-butyl-1-methylpyrrolidinium trifluorotris(pentafluoroethyl)phosphate & Pyr16 (C2F5)3PF3 \\
\hline 1-hexyl-1-methylpyrrolidinium bis(trifluoromethylsulfonyl)imide & Pyr16 (CF3SO2)2N \\
\hline
\end{tabular}

Cation hydrophobicity parameters $\log k_{\mathrm{c}, 0}$ were taken from [14] and [19]. The $\log k_{\mathrm{c}, 0}$ parameters for the cations 1-ethyl-3-methylimidazolium, 1-(3-hydroxypropyl)pyridinium, and 4-(ethoxymethyl)-4methylmorpholinium newly presented here were derived from capacity factors calculated from isocratic retention times on the same reversed phase HPLC column type (Polaris Ether C18 from Varian, $150 \mathrm{~mm}$, $3 \mathrm{~mm}$ inner diameter, $5 \mathrm{~m}$ particle size). Specifically, for each mobile phase composition, i.e. 2.5, 5, 10, 15 and $20 \%$ gradient grade acetonitrile complemented with $0.25 \%$ acetic acid (p.a., both Fluka, Buchs, Switzerland), a linear correlation was established between capacity factors under these conditions and previously established $[14,19] \log k_{\mathrm{c}, 0}$ values. For the three cations without such previously established values, the mean of the predictions based on these linear correlations was used as $\log k_{\mathrm{c}, 0}$ in Table 3 .

Determination of water solubility was carried out based on OECD guideline 105 for testing of chemicals. After establishment of approximate water solubility by a preliminary visual test, six aliquots of $100 \mathrm{~mL}$ water were equilibrated with the amount of ionic liquid resulting in five-fold oversaturation according to the preliminary result in a water bath at 30 degrees $\mathrm{C}$, and repeatedly mixed by thoroughly shaking them manually. After 24, 48, and 72 hours two aliquots were transferred to a water bath at 20 degrees $\mathrm{C}$. Each of those aliquots was gently centrifuged after a second $24 \mathrm{~h}$ equilibration period in the second water bath, and the ionic liquid concentration in the water phase was determined by reversed phase HPLC. The experimental uncertainty of the water solubility determinations depends on the soluble concentration and is better for ionic liquids exhibiting higher water solubility. Generally, deviations should be lower than $0.1 \log$ units. 
HPLC determination of ionic liquid concentrations in the saturated solutions was carried out using isocratic reversed phase HPLC on a Hewlett Packard 1100 Series system, equipped with online degasser, variable wavelength UV detector and a Bruker esquire ESI-MS ion trap detector. For the UV-absorbing cations, a Polaris Ether column from Varian also used for the hydrophobicity determination was used, with $20 \mathrm{mM} \mathrm{KH}_{2} \mathrm{PO}_{4}$ and $3.9 \mathrm{mM} \mathrm{H}_{3} \mathrm{PO}_{4}$ on channel A and gradient grade acetonitrile on channel $\mathrm{B}$, using a suitable percentage of acetonitrile for each cation, and UV detection at $212 \mathrm{~nm}$. For the cations without UV absorption, the same acetic acid/acetonitrile eluent system used in the hydrophobicity determination was used isocratically on the Polaris Ether RP 18 column, in order to have better compatibility with the ESI interface, again adapting the acetonitrile percentage to the analyte cation. For the quantification by ESI-MS, only a concentration range of two decades at maximum could be used, because of the nonlinear relation between concentration and peak area. In both cases, the $\mathrm{pH}$ of the eluent has to be around 3 , in order to avoid tailing and overly long retention times. Conversely, for the most hydrophilic cations, a MonoChrom MS column from Varian was used for quantification, in order to obtain sufficient retention.

Ionic liquid concentrations at saturation derived from cation analysis via HPLC-ESI-MS were double checked by determining peak areas of the hydrophobic anions bis(trifluoromethlysulfonyl)imide and trifluorotris(pentafluoroethyl)phosphate in the negative mode of the ion trap detector on the same column, but with only $0.1 \%$ to $0.25 \%$ acetic acid as the aqueous part and 60 to $70 \% \mathrm{MeOH}$. They diverged by less than ten percent, and the mean of cation and anion concentrations was taken as the final result.

\section{Results}

The data analysed using the model derived above are listed in Table 3. Most of the cation hydrophobicity data has already been published elsewhere by our group $[14,19]$. The water solubility data generated by us has not been published previously.

Table 3. Water solubility at temperatures within $293.15 \pm 5 \mathrm{~K}$ and cation hydrophobicity. Data without source attribution are published here for the first time. Data with a mole fraction solubility greater than 0.05 are not considered, because this would conflict with Assumptions 1 and 2 stated in the text. Data from Branco et al. [20] were not considered as they strongly diverge from other sources for an unknown reason.

\begin{tabular}{|c|c|c|c|c|c|c|}
\hline \multirow[b]{2}{*}{ Cation $^{[\mathrm{a}]}$} & \multicolumn{2}{|c|}{ Cation hydrophobicity } & \multirow[b]{2}{*}{ Anion $^{[b]}$} & \multicolumn{3}{|c|}{ IL water solubility } \\
\hline & $\log k_{0, \mathrm{c}}$ & & & $\log _{10} x_{\mathrm{IL}, \mathrm{w}}^{\mathrm{sat}}$ & $T[\mathrm{~K}]$ & \\
\hline IM16 & 1.2 & [14] & $\left(\mathrm{C}_{2} \mathrm{~F}_{5}\right)_{3} \mathrm{PF}_{3}$ & -5.93 & 293.15 & \\
\hline Pyr14 & 0.57 & [14] & $\left(\mathrm{C}_{2} \mathrm{~F}_{5}\right)_{3} \mathrm{PF}_{3}$ & -5.43 & 293.15 & \\
\hline Py8-4Me & 2 & [14] & $\left(\mathrm{C}_{2} \mathrm{~F}_{5} \mathrm{SO}_{2}\right)_{2} \mathrm{~N}$ & -5.4 & 298.15 & [21] \\
\hline Py8-4Me & 2 & [14] & $\left(\mathrm{CF}_{3} \mathrm{SO}_{2}\right)_{2} \mathrm{~N}$ & -5.09 & 298.15 & [21] \\
\hline IM16 & 1.2 & [14] & $\left(\mathrm{CF}_{3} \mathrm{SO}_{2}\right)_{3} \mathrm{C}$ & -5.04 & 293.15 & \\
\hline Py8-4Me & 2 & {$[14]$} & $\mathrm{AsF}_{6}$ & -4.91 & 298.15 & [21] \\
\hline Pyr18 & 1.9 & [14] & $\left(\mathrm{CF}_{3} \mathrm{SO}_{2}\right)_{2} \mathrm{~N}$ & -4.71 & 298 & [22] \\
\hline
\end{tabular}


Table 3. Cont.

\begin{tabular}{|c|c|c|c|c|c|c|}
\hline \multirow{3}{*}{$\begin{array}{l}\text { Cation }^{[\mathrm{a}]} \\
\text { IM18 }\end{array}$} & \multirow{2}{*}{\multicolumn{2}{|c|}{$\begin{array}{l}\text { Cation hydrophobicity } \\
\log k_{0, \mathrm{c}}\end{array}$}} & \multirow{3}{*}{$\frac{\text { Anion }^{[\mathrm{b}]}}{\left(\mathrm{C}_{2} \mathrm{~F}_{5} \mathrm{SO}_{2}\right)_{2} \mathrm{~N}}$} & \multicolumn{3}{|c|}{ IL water solubility } \\
\hline & & & & \multirow{2}{*}{$\begin{array}{c}\log _{10} x_{\mathrm{w}}^{\mathrm{sat}} \\
-4.7\end{array}$} & \multicolumn{2}{|c|}{$T[\mathrm{~K}]$} \\
\hline & 1.9 & {$[14]$} & & & 298 & [23] \\
\hline Py8-4Me & 2 & [14] & $\left(\mathrm{C}_{4} \mathrm{~F}_{9}\right) \mathrm{SO}_{3}$ & -4.63 & 298.15 & [21] \\
\hline IM18 & 1.9 & {$[14]$} & $\left(\mathrm{CF}_{3} \mathrm{SO}_{2}\right)_{2} \mathrm{~N}$ & -4.6 & 298 & [23] \\
\hline IM18 & 1.9 & {$[14]$} & $\left(\mathrm{CF}_{3} \mathrm{SO}_{2}\right)_{2} \mathrm{~N}$ & -4.59 & 293.15 & \\
\hline Py6-4NMe2 & 1.8 & [19] & $\left(\mathrm{CF}_{3} \mathrm{SO}_{2}\right)_{2} \mathrm{~N}$ & -4.53 & 293.15 & \\
\hline Py6-4NMe2 & 1.8 & [19] & $\left(\mathrm{CF}_{3} \mathrm{SO}_{2}\right)_{2} \mathrm{~N}$ & -4.53 & 296.5 & [24] \\
\hline IM18 & 1.9 & {$[14]$} & $\left(\mathrm{CF}_{3} \mathrm{SO}_{2}\right)_{2} \mathrm{~N}$ & -4.5 & 288.15 & [25] \\
\hline IM18 & 1.9 & {$[14]$} & $\left(\mathrm{CF}_{3} \mathrm{SO}_{2}\right)_{2} \mathrm{~N}$ & -4.49 & 293.15 & [25] \\
\hline IM18 & 1.9 & [14] & $\left(\mathrm{CF}_{3} \mathrm{SO}_{2}\right)_{2} \mathrm{~N}$ & -4.47 & 298.15 & {$[25]$} \\
\hline IM14 & 0.67 & [14] & $\left(\mathrm{CF}_{3} \mathrm{SO}_{2}\right)_{3} \mathrm{C}$ & -4.44 & 296.5 & [24] \\
\hline IM17 & 1.6 & [14] & $\left(\mathrm{CF}_{3} \mathrm{SO}_{2}\right)_{2} \mathrm{~N}$ & -4.31 & 288.15 & [25] \\
\hline IM17 & 1.6 & [14] & $\left(\mathrm{CF}_{3} \mathrm{SO}_{2}\right)_{2} \mathrm{~N}$ & -4.3 & 293.15 & [25] \\
\hline IM17 & 1.6 & {$[14]$} & $\left(\mathrm{CF}_{3} \mathrm{SO}_{2}\right)_{2} \mathrm{~N}$ & -4.29 & 298.15 & [25] \\
\hline IM18 & 1.9 & {$[14]$} & $\left(\mathrm{C}_{4} \mathrm{~F}_{9}\right) \mathrm{SO}_{3}$ & -4.23 & 298 & [26] \\
\hline IM16 & 1.2 & {$[14]$} & $\left(\mathrm{CF}_{3} \mathrm{SO}_{2}\right)_{2} \mathrm{~N}$ & -4.18 & 293.15 & \\
\hline IM16-2Me & 1.4 & [14] & $\left(\mathrm{CF}_{3} \mathrm{SO}_{2}\right)_{2} \mathrm{~N}$ & -4.15 & 296.5 & [24] \\
\hline IM18 & 1.9 & [14] & $\left(\mathrm{CF}_{3} \mathrm{SO}_{2}\right)_{2} \mathrm{~N}$ & -4.14 & 296.5 & [24] \\
\hline Pyr16 & 1.2 & [14] & $\left(\mathrm{CF}_{3} \mathrm{SO}_{2}\right)_{2} \mathrm{~N}$ & -4.12 & 293.15 & \\
\hline IM18 & 1.9 & {$[14]$} & $\left(\mathrm{CF}_{3} \mathrm{SO}_{2}\right)_{2} \mathrm{~N}$ & -4.1 & 298 & [26] \\
\hline IM18 & 1.9 & [14] & $\left(\mathrm{CF}_{3} \mathrm{SO}_{2}\right)_{2} \mathrm{~N}$ & -4.1 & 293.15 & [27] \\
\hline IM16 & 1.2 & [14] & $\left(\mathrm{CF}_{3} \mathrm{SO}_{2}\right)_{2} \mathrm{~N}$ & -4.05 & 288.15 & {$[25]$} \\
\hline IM16 & 1.2 & {$[14]$} & $\left(\mathrm{CF}_{3} \mathrm{SO}_{2}\right)_{2} \mathrm{~N}$ & -4.05 & 293.15 & [25] \\
\hline IM16 & 1.2 & [14] & $\left(\mathrm{CF}_{3} \mathrm{SO}_{2}\right)_{2} \mathrm{~N}$ & -4.03 & 296.5 & [24] \\
\hline IM16 & 1.2 & [14] & $\left(\mathrm{CF}_{3} \mathrm{SO}_{2}\right)_{2} \mathrm{~N}$ & -4.02 & 298.15 & [25] \\
\hline IM18 & 1.9 & [14] & $\mathrm{PF}_{6}$ & -3.95 & 288.15 & [28] \\
\hline IM18 & 1.9 & {$[14]$} & $\mathrm{PF}_{6}$ & -3.93 & 293.15 & \\
\hline IM18 & 1.9 & [14] & $\mathrm{PF}_{6}$ & -3.92 & 293.15 & {$[28]$} \\
\hline IM18 & 1.9 & [14] & $\mathrm{PF}_{6}$ & -3.9 & 298.15 & {$[28]$} \\
\hline IM16 & 1.2 & [14] & $\left(\mathrm{CF}_{3} \mathrm{SO}_{2}\right)_{2} \mathrm{~N}$ & -3.86 & 293.15 & [27] \\
\hline Pip14 & 0.68 & [19] & $\left(\mathrm{CF}_{3} \mathrm{SO}_{2}\right)_{2} \mathrm{~N}$ & -3.78 & 298 & [22] \\
\hline IM15 & 0.92 & {$[14]$} & $\left(\mathrm{CF}_{3} \mathrm{SO}_{2}\right)_{2} \mathrm{~N}$ & -3.74 & 288.15 & [25] \\
\hline IM15 & 0.92 & {$[14]$} & $\left(\mathrm{CF}_{3} \mathrm{SO}_{2}\right)_{2} \mathrm{~N}$ & -3.73 & 293.15 & [25] \\
\hline IM15 & 0.92 & {$[14]$} & $\left(\mathrm{CF}_{3} \mathrm{SO}_{2}\right)_{2} \mathrm{~N}$ & -3.71 & 298.15 & [25] \\
\hline Py4-3Me & 0.73 & [14] & $\left(\mathrm{CF}_{3} \mathrm{SO}_{2}\right)_{2} \mathrm{~N}$ & -3.7 & 296.5 & [24] \\
\hline Py4-4Me & 0.73 & [14] & $\left(\mathrm{CF}_{3} \mathrm{SO}_{2}\right)_{2} \mathrm{~N}$ & -3.69 & 298 & [26] \\
\hline Py8-4Me & 2 & [14] & $\mathrm{PhBF}_{3}$ & -3.6 & 298.15 & [21] \\
\hline
\end{tabular}


Table 3. Cont.

\begin{tabular}{|c|c|c|c|c|c|c|}
\hline \multirow[b]{2}{*}{ Cation $^{[\mathrm{a}]}$} & \multicolumn{2}{|c|}{ Cation hydrophobicity } & \multirow[b]{2}{*}{ Anion $^{[b]}$} & \multicolumn{3}{|c|}{ IL water solubility } \\
\hline & $\log k_{0, \mathrm{c}}$ & & & $\log _{10} x_{\mathrm{w}}^{\mathrm{sat}}$ & $T[\mathrm{~K}]$ & \\
\hline Pyr14 & 0.57 & [14] & $\left(\mathrm{CF}_{3} \mathrm{SO}_{2}\right)_{2} \mathrm{~N}$ & -3.59 & 298 & [22] \\
\hline Pyr14 & 0.57 & [14] & $\left(\mathrm{CF}_{3} \mathrm{SO}_{2}\right)_{2} \mathrm{~N}$ & -3.57 & 293.15 & \\
\hline IM14 & 0.67 & {$[14]$} & $\left(\mathrm{CF}_{3} \mathrm{SO}_{2}\right)_{2} \mathrm{~N}$ & -3.54 & 288.15 & [25] \\
\hline IM14 & 0.67 & [14] & $\left(\mathrm{CF}_{3} \mathrm{SO}_{2}\right)_{2} \mathrm{~N}$ & -3.53 & 293.15 & [25] \\
\hline IM14 & 0.67 & [14] & $\left(\mathrm{CF}_{3} \mathrm{SO}_{2}\right)_{2} \mathrm{~N}$ & -3.51 & 298.15 & [25] \\
\hline IM14 & 0.67 & [14] & $\left(\mathrm{CF}_{3} \mathrm{SO}_{2}\right)_{2} \mathrm{~N}$ & -3.51 & 298 & [26] \\
\hline IM14 & 0.67 & {$[14]$} & $\left(\mathrm{CF}_{3} \mathrm{SO}_{2}\right)_{2} \mathrm{~N}$ & -3.5 & 296.5 & [24] \\
\hline IM14 & 0.67 & [14] & $\left(\mathrm{CF}_{3} \mathrm{SO}_{2}\right)_{2} \mathrm{~N}$ & -3.5 & 293.15 & \\
\hline IM14 & 0.67 & [14] & $\left(\mathrm{CF}_{3} \mathrm{SO}_{2}\right)_{2} \mathrm{~N}$ & -3.49 & 294.15 & [29] \\
\hline IM14 & 0.67 & [14] & $\left(\mathrm{CF}_{3} \mathrm{SO}_{2}\right)_{2} \mathrm{~N}$ & -3.46 & 293.15 & [27] \\
\hline IM18 & 1.9 & [14] & $\mathrm{PF}_{6}$ & -3.46 & 295 & [30] \\
\hline IM16 & 1.2 & [14] & $\mathrm{PF}_{6}$ & -3.45 & 288.15 & [28] \\
\hline IM16 & 1.2 & {$[14]$} & $\mathrm{PF}_{6}$ & -3.41 & 293.15 & [28] \\
\hline IM16 & 1.2 & {$[14]$} & $\mathrm{PF}_{6}$ & -3.36 & 298.15 & [28] \\
\hline IM16 & 1.2 & [14] & $\mathrm{PF}_{6}$ & -3.35 & 293.15 & \\
\hline IM13 & 0.42 & [14] & $\left(\mathrm{CF}_{3} \mathrm{SO}_{2}\right)_{2} \mathrm{~N}$ & -3.29 & 288.15 & [25] \\
\hline IM13 & 0.42 & [14] & $\left(\mathrm{CF}_{3} \mathrm{SO}_{2}\right)_{2} \mathrm{~N}$ & -3.28 & 293.15 & [25] \\
\hline IM13 & 0.42 & [14] & $\left(\mathrm{CF}_{3} \mathrm{SO}_{2}\right)_{2} \mathrm{~N}$ & -3.27 & 298.15 & [25] \\
\hline Mor11O2 & 0.17 & & $\left(\mathrm{CF}_{3} \mathrm{SO}_{2}\right)_{2} \mathrm{~N}$ & -3.19 & 293.15 & \\
\hline IM14 & 0.67 & [14] & $\left(\mathrm{C}_{4} \mathrm{~F}_{9}\right) \mathrm{SO}_{3}$ & -3.15 & 298 & [26] \\
\hline IM12 & 0.22 & & $\left(\mathrm{CF}_{3} \mathrm{SO}_{2}\right)_{2} \mathrm{~N}$ & -3.12 & 288.15 & [25] \\
\hline IM12 & 0.22 & & $\left(\mathrm{CF}_{3} \mathrm{SO}_{2}\right)_{2} \mathrm{~N}$ & -3.1 & 296.5 & [24] \\
\hline IM12 & 0.22 & & $\left(\mathrm{CF}_{3} \mathrm{SO}_{2}\right)_{2} \mathrm{~N}$ & -3.1 & 293.15 & [25] \\
\hline Py8-4Me & 2 & [14] & $\mathrm{CF}_{3} \mathrm{SO}_{3}$ & -3.09 & 298.15 & {$[21]$} \\
\hline IM12 & 0.22 & & $\left(\mathrm{CF}_{3} \mathrm{SO}_{2}\right)_{2} \mathrm{~N}$ & -3.08 & 293.15 & [27] \\
\hline IM12 & 0.22 & & $\left(\mathrm{CF}_{3} \mathrm{SO}_{2}\right)_{2} \mathrm{~N}$ & -3.08 & 298.15 & [25] \\
\hline Py4-4Me & 0.73 & {$[14]$} & $\left(\mathrm{C}_{4} \mathrm{~F}_{9}\right) \mathrm{SO}_{3}$ & -3.03 & 298 & [26] \\
\hline IM14 & 0.67 & [14] & $\mathrm{PF}_{6}$ & -3 & 288.15 & [28] \\
\hline Py8-4Me & 2 & [14] & $\mathrm{BF}_{4}$ & -2.98 & 298.15 & [21] \\
\hline IM14 & 0.67 & {$[14]$} & $\mathrm{PF}_{6}$ & -2.96 & 293.15 & [28] \\
\hline IM14 & 0.67 & {$[14]$} & $\mathrm{PF}_{6}$ & -2.93 & 294 & [31] \\
\hline IM18 & 1.9 & {$[14]$} & $\mathrm{BF}_{4}$ & -2.93 & 295 & [30] \\
\hline IM14 & 0.67 & [14] & $\mathrm{PF}_{6}$ & -2.92 & 298.15 & [28] \\
\hline IM14 & 0.67 & [14] & $\mathrm{PF}_{6}$ & -2.9 & 293.15 & \\
\hline IM14 & 0.67 & [14] & $\mathrm{PF}_{6}$ & -2.9 & 296.5 & [24] \\
\hline IM14 & 0.67 & [14] & $\mathrm{PF}_{6}$ & -2.89 & 295 & [30] \\
\hline
\end{tabular}


Table 3. Cont.

\begin{tabular}{|c|c|c|c|c|c|c|}
\hline \multirow[b]{2}{*}{ Cation $^{[\mathrm{a}]}$} & \multicolumn{2}{|c|}{ Cation hydrophobicity } & \multirow[b]{2}{*}{ Anion $^{[b]}$} & \multicolumn{3}{|c|}{ IL water solubility } \\
\hline & $\log k_{0, \mathrm{c}}$ & & & $\log _{10} x_{\mathrm{w}}^{\mathrm{sat}}$ & $T[\mathrm{~K}]$ & \\
\hline IM14 & 0.67 & {$[14]$} & $\mathrm{PF}_{6}$ & -2.87 & 294.15 & [29] \\
\hline IM14 & 0.67 & [14] & $\mathrm{PF}_{6}$ & -2.8 & 293.15 & [27] \\
\hline IM12 & 0.22 & & $\mathrm{~B}(\mathrm{CN})_{4}$ & -2.46 & 296.5 & {$[24]$} \\
\hline Рy3OH & -0.09 & & $\left(\mathrm{CF}_{3} \mathrm{SO}_{2}\right)_{2} \mathrm{~N}$ & -2.43 & 293.15 & \\
\hline IM12OH & -0.28 & [19] & $\left(\mathrm{CF}_{3} \mathrm{SO}_{2}\right)_{2} \mathrm{~N}$ & -2.34 & 296.5 & [24] \\
\hline N4444 & 2.3 & [14] & $(6-2 \mathrm{Et}) 2 \mathrm{SS}$ & -1.52 & 298 & {$[32]$} \\
\hline
\end{tabular}

[a] Cation acronyms explained in Table 1

[b] (6-2Et)-2SS = bis(2-ethylhexyl)sulfosuccinate

In Figure 2, water solubilities of ionic liquids that form a second phase with water at room temperature are plotted against the hydrophobicity of their cation. The trend lines in the graph resulting from fitting the linear model specified in equation 1 . Only data for anions combined with at least two different cations are shown.

The two available water solubility data for the $\left(\mathrm{C}_{2} \mathrm{~F}_{5} \mathrm{SO}_{2}\right)_{2} \mathrm{~N}$ anion diverge by 0.7 log units, despite the similar hydrophobicity parameters of the two cations (Py8-4Me and IM18). Similar, but smaller deviations are found for the $\left(\mathrm{C}_{2} \mathrm{~F}_{5} \mathrm{SO}_{2}\right)_{2} \mathrm{~N}$ salts of the same cations. It would be tempting to attribute these differences to H-bonding between the hydrogen in 2-position of the imidazolium ring and the sulfoxy groups of the bis(perfluoroalkylsulfonyl)imides in the pure ionic liquid, leading to smaller water solubilities of the imidazolium bis(perfluoroalkylsulfonyl)imides than predicted by their cation hydrophobicities. However, the size of the intralaboratory variability of the data as apparent in Table 5 in the Appendix does not really allow for such a conclusion, given that these data were generated by different groups.

In Table 4, the statistical parameters resulting from fitting this model to two sets of data are given. When modeling only the water solubility data from our labs (UFT only), the RSE was a little smaller than in the model for all solubility data (Full). This is not really surprising, as the equilibration procedure can have an impact on the results. The fact that the slope parameter $m$ approximates the value expected according to the theoretical considerations is a striking evidence that both partitioning processes (ionic liquid/water equilibrium and cation partitioning in the HPLC column) are governed by the same cation property, even though the second phase is an ionic liquid in the one case, and a hydrophobic interphase at the surface of the chemically modified silica particles in the other case. The most plausible explanation is indeed to assume that this common property is their activity coefficient in aqueous systems with the pure ionic liquid as the reference state. 
Figure 2. Plot of water solubility against $\log k_{0, c}$ of the cation of various ionic liquids that form a second phase with water at room temperature. To the left, only water solubility data generated in our labs are presented, to the right, water solubility data from the peer reviewed literature are included. Regression lines for constant anions have identical slopes $-m$ as defined by model equation 1. Only data for anions with solubility data for at least two different cations are plotted.

UFT data only

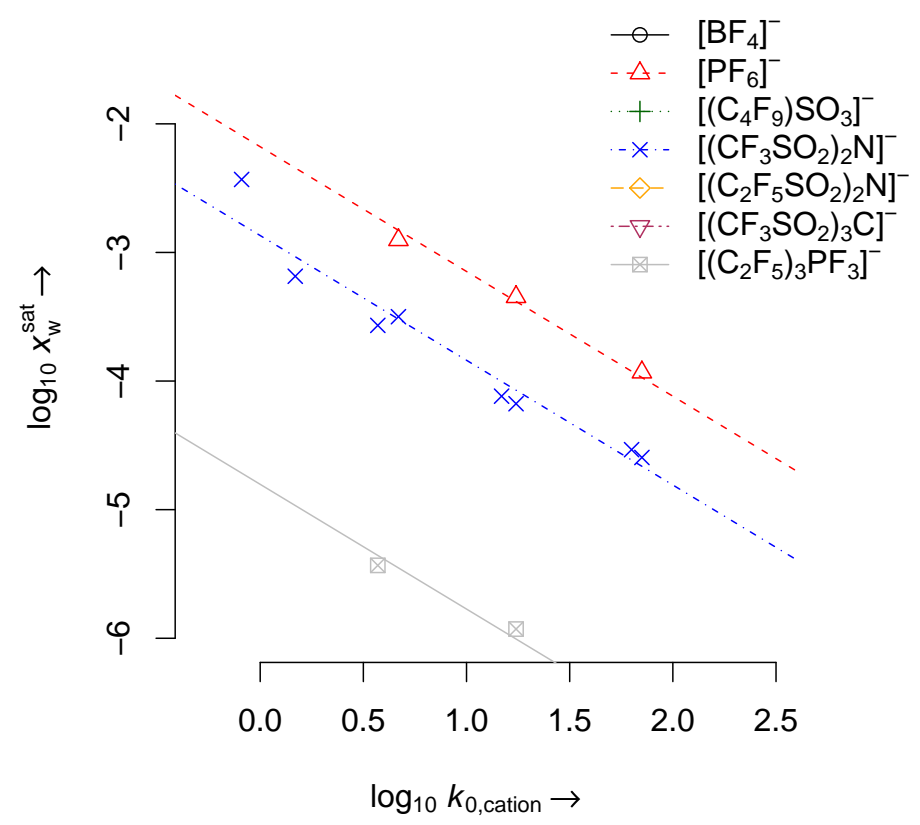

Full dataset

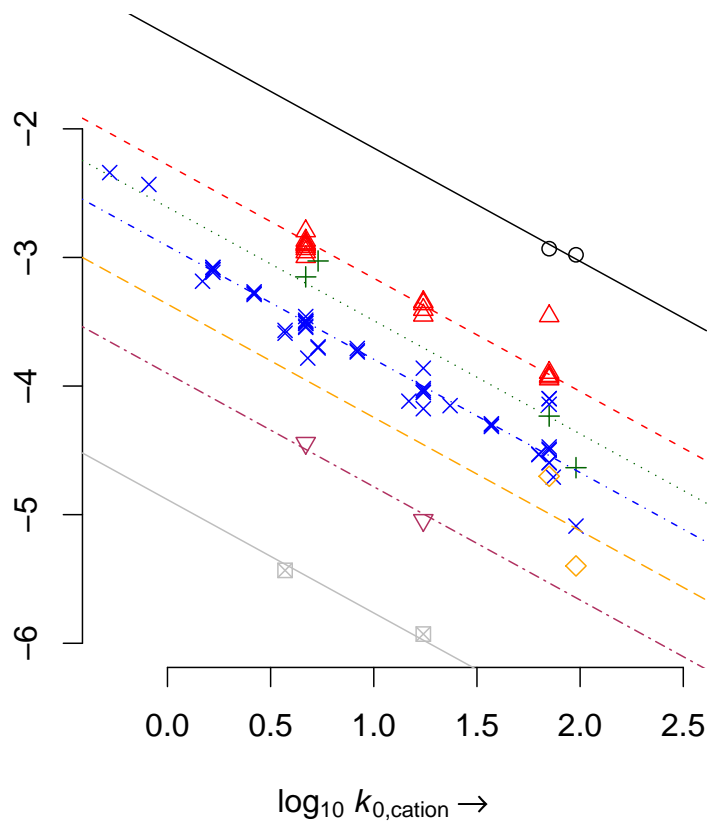

For the UFT only model, the slope $m$ is 0.97 , which is within the expected uncertainty for such a model. The larger deviation of $m$ from unity in the full model can partly be explained by a large scatter of experimental values for different 1-octyl-3-methylimidazolium ionic liquids. The lower values better fit the theory, which might be caused by a disturbance of the chosen analytical method by the formation of microdroplets, which is also a known problem in the determination of 1-octanol/water partition coefficients.

The anion constants $c_{a}$ in Table 4 can be seen as hydrophobicity measures, complementary to the $\log k_{0, \mathrm{c}}$ values for the cations. The fact that many of these constants are derived from data for only one ionic liquid explains the very high coefficient of determination $R^{2}$ for both models. It is worthy to note that the anion constants in Table 4 for the $\left[\mathrm{PF}_{6}\right]^{-},\left[\left(\mathrm{CF}_{3} \mathrm{SO}_{2}\right)_{2} \mathrm{~N}\right]^{-}$, and $\left[\left(\mathrm{CF}_{3} \mathrm{SO}_{2}\right)_{3} \mathrm{C}\right]^{-}$anions are very similar, regardless if only the UFT data are employed in the model building, or if they are supplemented by the more abundant and completely independent literature data. 
Table 4. Statistical parameters of the model described in equation 1 applied to only our own water solubility data at $293.15 \mathrm{~K}$ (UFT data only), and the complete water solubility dataset for $293.15 \pm 5 \mathrm{~K}$ (Full dataset) given in Table 3. Numbers in parentheses after the anion constants are the number of data points for each anion that the constant is based on. $n$ is the overall number of data points in the model, $\mathrm{df}$ is the number of statistical degrees of freedom, $R^{2}$ is the fraction of the variability in water solubility explained by the model, and RSE is the standard error of the residuals. Note that data points for anions that are only present in one IL have a zero residual, giving a favorable bias to $R^{2}$ and RSE. The anion acronym (6-2Et)2SS stands for bis(2-ethylhexyl)sulfosuccinate.

\begin{tabular}{|c|c|c|}
\hline Parameter & UFT data only & Full dataset \\
\hline$m$ & 0.97 & 0.881 \\
\hline$c_{(6-2 \mathrm{Et}) 2 \mathrm{SS}}$ & & $0.521(1)$ \\
\hline$c_{\mathrm{BF} 4}$ & & $-1.268(2)$ \\
\hline${ }^{c} \mathrm{CF} 3 \mathrm{SO} 3$ & & $-1.343(1)$ \\
\hline$c_{\mathrm{C}(\mathrm{CN}) 3}$ & & $-1.642(3)$ \\
\hline$c_{\mathrm{PhBF} 3}$ & & $-1.853(1)$ \\
\hline$c_{\mathrm{B}(\mathrm{CN}) 4}$ & & $-2.264(1)$ \\
\hline$c_{\mathrm{PF} 6}$ & $-2.178(3)$ & $-2.28(18)$ \\
\hline$c_{(\mathrm{C} 4 \mathrm{~F} 9) \mathrm{SO} 3}$ & & $-2.61(4)$ \\
\hline$c_{(\mathrm{CF} 3 \mathrm{SO} 2) 2 \mathrm{~N}}$ & $-2.868(8)$ & $-2.911(50)$ \\
\hline$c_{\mathrm{AsF} 6}$ & & $-3.165(1)$ \\
\hline$c_{(\mathrm{C} 2 \mathrm{~F} 5 \mathrm{SO} 2) 2 \mathrm{~N}}$ & & $-3.363(2)$ \\
\hline$c_{(\mathrm{CF} 3 \mathrm{SO} 2) 3 \mathrm{C}}$ & $-3.841(1)$ & $-3.902(2)$ \\
\hline$c_{(\mathrm{C} 2 \mathrm{~F} 5) 3 \mathrm{PF} 3}$ & $-4.803(2)$ & $-4.883(2)$ \\
\hline$n$ & 14 & 88 \\
\hline df & 9 & 74 \\
\hline$R^{2}$ & 0.999 & 0.998 \\
\hline RSE & 0.157 & 0.16 \\
\hline
\end{tabular}

A more direct determination of the anion hydrophobicity using an HPLC based method similar to the method of cation hydrophobicity determination used here was attempted. So far, the results have been found to be unreliable, presumably because of the limited suitability of the chromatographic partitioning system used.

The correlations presented here confound the validity of the $\log k_{0, \mathrm{c}}$ values as derived from HPLC as a measure of the tendency of a cation to evade aqueous phases. Previously, we have shown that the cytotoxicity of ionic liquids can be described by the cation $\log k_{0, \mathrm{c}}$ with an RSE value of 0.47 [14]. The much larger RSE in that former study can be attributed to a) the fact that several anions were included in 
one correlation, b) to the inclusion of the large aromatic quinolinium cations in the earlier study, and c) to the fact that biological test systems generally yield higher variabilities than physicochemical systems.

The applicability domain of the model for predicting water solubilities from this model is naturally restricted by the availability of $\log k_{0, \mathrm{c}}$ values (see [16] and this study) and anion constants (Table 4). The accuracy of $\log k_{0, \mathrm{c}}$ values is best for $0<\log k_{0, \mathrm{c}}<4$, because of limitations of the determination method. In this interval, the uncertainty is estimated to be less than $0.1 \log$ units. The estimated standard error of predictions of water solubility in this interval is $0.2 \mathrm{log}$ units for the seven anions shown on the right side of Figure 2 (model for selected data). For the anions with only one data point, we advise to only extrapolate to ionic liquids with cations that have a $\log k_{0, \mathrm{c}}$ that does not deviate more than $1 \log$ unit from the $\log k_{0, c}$ of the given data point.

For the convenience of the reader, the water solubilities estimated by the full solubility model are listed together with the experimental data in Table 5 in the Appendix. Besides the explanations given above, no systematic deviations from the model were noted. In general, the database for the bis(trifluoromethylsulfonyl) imide ionic liquids is quite extensive, and there is a very good agreement between the majority of literature sources where more than one value was found for the same ionic liquid. However, there are several outlying data points e.g. for IM18 bis(trifluoromethylsulfonyl)imide, that can only be explained by a considerable interlaboratory variability.

The approach taken here is in some ways similar to the one taken by Kakiuchi and co-workers, explaining the water solubility of ionic liquids in terms of their ion transfer potentials between ionic liquid and water [23], for which the ion transfer potentials between nitrobenzene and water are proposed as an approximation [33]. This group provided experimental evidence for the assumption that the ion transfer potentials between ionic liquids and water for the hydrophobic ions is independent of the concentration of hydrophilic ions like $\mathrm{Na}, \mathrm{K}$ and $\mathrm{Cl}$ in the aqueous phase. This can be taken as a corroboration of our assumption, that the activity coefficients of the hydrophobic cations are similar in water as in the mobile phase of our liquid chromatographic system (Assumption 7 in the supporting information). However, the reliability of prediction methods for the water solubility of ionic liquids based on such theories is limited by the quantity and precision of experimental data.

\section{Conclusions}

A new pathway to the approximate quantification of aqueous ion solvation is shown, making use of the relative weakness of interactions between ionic liquid ions as compared to their hydrophobicities. We believe that the excess free energies of IL solution in aqueous phases that can be estimated from $\log k_{0, \mathrm{c}}$ and $c_{\mathrm{A}}$ values presented here are a valuable tool to quantitatively predict equilibrium concentrations of ionic liquids in aqueous phases, contributing to the toolbox necessary for a molecular design of ionic liquid applications as well as to a more thorough understanding of their partitioning behavior in general.

\section{Acknowledgements}

This work was partially funded by the German Federal Ministry of Education and Research (bmb+f), project grant "Praktikable asymmetrische Reaktionen durch Biokatalyse: Integrierte Prozesse mit ionischen Flüssigkeiten zur Gewinnung chiraler Alkohole". Support from Merck KGaA including the supply 
of ionic liquids within a strategic partnership is gratefully acknowledged. A. Othman is grateful to the scholarship provided by Mohamed Farid Khamis Foundation (MFK) which made it possible for him to share in this work. We would like to thank Prof. J. Thöming for many helpful comments on earlier versions of the manuscript. We also thank the anonymous reviewers for their valuable comments and suggestions.

\section{Appendix}

Table 5. Overview of experimental (superscript exp) and estimated (superscript est) water solubility data, sorted by the estimated values. $\Delta_{\text {est,exp }}$ is the difference between estimated and observed values on a log scale. The model based on all suitable data was used for the estimation.

\begin{tabular}{|c|c|c|c|c|c|c|}
\hline Cation $^{[a]}$ & Anion $^{[b]}$ & $\log _{10} x$ & $\begin{array}{l}\text { sat,exp } \\
\text { IL,w }\end{array}$ & $\log _{10} x_{\mathrm{IL}, \mathrm{w}}^{\mathrm{sat}, \mathrm{est}}$ & $\Delta_{\text {est,exp }}$ & Temperature $[\mathrm{K}]$ \\
\hline IM16 & $\left(\mathrm{C}_{2} \mathrm{~F}_{5}\right)_{3} \mathrm{PF}_{3}$ & -5.93 & & -5.98 & -0.05 & 293.15 \\
\hline Pyr14 & $\left(\mathrm{C}_{2} \mathrm{~F}_{5}\right)_{3} \mathrm{PF}_{3}$ & -5.43 & & -5.39 & 0.04 & 293.15 \\
\hline Py8-4Me & $\left(\mathrm{C}_{2} \mathrm{~F}_{5} \mathrm{SO}_{2}\right)_{2} \mathrm{~N}$ & -5.4 & {$[21]$} & -5.11 & 0.29 & 298.15 \\
\hline IM16 & $\left(\mathrm{CF}_{3} \mathrm{SO}_{2}\right)_{3} \mathrm{C}$ & -5.04 & & -4.99 & 0.05 & 293.15 \\
\hline IM18 & $\left(\mathrm{C}_{2} \mathrm{~F}_{5} \mathrm{SO}_{2}\right)_{2} \mathrm{~N}$ & -4.7 & [23] & -4.99 & -0.29 & 298 \\
\hline Py8-4Me & $\mathrm{AsF}_{6}$ & -4.91 & [21] & -4.91 & 0 & 298.15 \\
\hline Py8-4Me & $\left(\mathrm{CF}_{3} \mathrm{SO}_{2}\right)_{2} \mathrm{~N}$ & -5.09 & [21] & -4.66 & 0.43 & 298.15 \\
\hline Pyr18 & $\left(\mathrm{CF}_{3} \mathrm{SO}_{2}\right)_{2} \mathrm{~N}$ & -4.71 & {$[22]$} & -4.56 & 0.15 & 298 \\
\hline IM18 & $\left(\mathrm{CF}_{3} \mathrm{SO}_{2}\right)_{2} \mathrm{~N}$ & -4.5 & {$[25]$} & -4.54 & -0.04 & 288.15 \\
\hline IM18 & $\left(\mathrm{CF}_{3} \mathrm{SO}_{2}\right)_{2} \mathrm{~N}$ & -4.59 & & -4.54 & 0.05 & 293.15 \\
\hline IM18 & $\left(\mathrm{CF}_{3} \mathrm{SO}_{2}\right)_{2} \mathrm{~N}$ & -4.1 & [27] & -4.54 & -0.44 & 293.15 \\
\hline IM18 & $\left(\mathrm{CF}_{3} \mathrm{SO}_{2}\right)_{2} \mathrm{~N}$ & -4.49 & {$[25]$} & -4.54 & -0.05 & 293.15 \\
\hline IM18 & $\left(\mathrm{CF}_{3} \mathrm{SO}_{2}\right)_{2} \mathrm{~N}$ & -4.14 & {$[24]$} & -4.54 & -0.4 & 296.5 \\
\hline IM18 & $\left(\mathrm{CF}_{3} \mathrm{SO}_{2}\right)_{2} \mathrm{~N}$ & -4.1 & [26] & -4.54 & -0.44 & 298 \\
\hline IM18 & $\left(\mathrm{CF}_{3} \mathrm{SO}_{2}\right)_{2} \mathrm{~N}$ & -4.6 & [23] & -4.54 & 0.06 & 298 \\
\hline IM18 & $\left(\mathrm{CF}_{3} \mathrm{SO}_{2}\right)_{2} \mathrm{~N}$ & -4.47 & {$[25]$} & -4.54 & -0.07 & 298.15 \\
\hline Py6-4NMe2 & $\left(\mathrm{CF}_{3} \mathrm{SO}_{2}\right)_{2} \mathrm{~N}$ & -4.53 & & -4.5 & 0.03 & 293.15 \\
\hline Py6-4NMe2 & $\left(\mathrm{CF}_{3} \mathrm{SO}_{2}\right)_{2} \mathrm{~N}$ & -4.53 & [24] & -4.5 & 0.03 & 296.5 \\
\hline IM14 & $\left(\mathrm{CF}_{3} \mathrm{SO}_{2}\right)_{3} \mathrm{C}$ & -4.44 & [24] & -4.49 & -0.05 & 296.5 \\
\hline Py8-4Me & $\left(\mathrm{C}_{4} \mathrm{~F}_{9}\right) \mathrm{SO}_{3}$ & -4.63 & [21] & -4.35 & 0.28 & 298.15 \\
\hline IM17 & $\left(\mathrm{CF}_{3} \mathrm{SO}_{2}\right)_{2} \mathrm{~N}$ & -4.31 & {$[25]$} & -4.29 & 0.02 & 288.15 \\
\hline IM17 & $\left(\mathrm{CF}_{3} \mathrm{SO}_{2}\right)_{2} \mathrm{~N}$ & -4.3 & {$[25]$} & -4.29 & 0.01 & 293.15 \\
\hline IM17 & $\left(\mathrm{CF}_{3} \mathrm{SO}_{2}\right)_{2} \mathrm{~N}$ & -4.29 & {$[25]$} & -4.29 & 0 & 298.15 \\
\hline IM18 & $\left(\mathrm{C}_{4} \mathrm{~F}_{9}\right) \mathrm{SO}_{3}$ & -4.23 & [26] & -4.24 & -0.01 & 298 \\
\hline IM16-2Me & $\left(\mathrm{CF}_{3} \mathrm{SO}_{2}\right)_{2} \mathrm{~N}$ & -4.15 & [24] & -4.12 & 0.03 & 296.5 \\
\hline IM16 & $\left(\mathrm{CF}_{3} \mathrm{SO}_{2}\right)_{2} \mathrm{~N}$ & -4.05 & [25] & -4 & 0.05 & 288.15 \\
\hline
\end{tabular}


Table 5. Cont.

\begin{tabular}{|c|c|c|c|c|c|c|}
\hline Cation $^{[\mathrm{a}]}$ & Anion $^{[b]}$ & \multicolumn{2}{|c|}{$\log _{10} x_{\mathrm{IL}, \mathrm{w}}^{\text {sat,exp }}$} & $\log _{10} x_{\mathrm{IL}, \mathrm{w}}^{\mathrm{sat}, \mathrm{est}}$ & $\Delta_{\text {est,exp }}$ & Temperature $[\mathrm{K}]$ \\
\hline IM16 & $\left(\mathrm{CF}_{3} \mathrm{SO}_{2}\right)_{2} \mathrm{~N}$ & -4.18 & & -4 & 0.18 & 293.15 \\
\hline IM16 & $\left(\mathrm{CF}_{3} \mathrm{SO}_{2}\right)_{2} \mathrm{~N}$ & -3.86 & [27] & -4 & -0.14 & 293.15 \\
\hline IM16 & $\left(\mathrm{CF}_{3} \mathrm{SO}_{2}\right)_{2} \mathrm{~N}$ & -4.05 & [25] & -4 & 0.05 & 293.15 \\
\hline IM16 & $\left(\mathrm{CF}_{3} \mathrm{SO}_{2}\right)_{2} \mathrm{~N}$ & -4.03 & [24] & -4 & 0.03 & 296.5 \\
\hline IM16 & $\left(\mathrm{CF}_{3} \mathrm{SO}_{2}\right)_{2} \mathrm{~N}$ & -4.02 & [25] & -4 & 0.02 & 298.15 \\
\hline Pyr16 & $\left(\mathrm{CF}_{3} \mathrm{SO}_{2}\right)_{2} \mathrm{~N}$ & -4.12 & & -3.94 & 0.18 & 293.15 \\
\hline IM18 & $\mathrm{PF}_{6}$ & -3.95 & [28] & -3.91 & 0.04 & 288.15 \\
\hline IM18 & $\mathrm{PF}_{6}$ & -3.92 & {$[28]$} & -3.91 & 0.01 & 293.15 \\
\hline IM18 & $\mathrm{PF}_{6}$ & -3.93 & & -3.91 & 0.02 & 293.15 \\
\hline IM18 & $\mathrm{PF}_{6}$ & -3.46 & {$[30]$} & -3.91 & -0.45 & 295 \\
\hline IM18 & $\mathrm{PF}_{6}$ & -3.9 & [28] & -3.91 & -0.01 & 298.15 \\
\hline IM15 & $\left(\mathrm{CF}_{3} \mathrm{SO}_{2}\right)_{2} \mathrm{~N}$ & -3.74 & [25] & -3.72 & 0.02 & 288.15 \\
\hline IM15 & $\left(\mathrm{CF}_{3} \mathrm{SO}_{2}\right)_{2} \mathrm{~N}$ & -3.73 & [25] & -3.72 & 0.01 & 293.15 \\
\hline IM15 & $\left(\mathrm{CF}_{3} \mathrm{SO}_{2}\right)_{2} \mathrm{~N}$ & -3.71 & {$[25]$} & -3.72 & -0.01 & 298.15 \\
\hline Py8-4Me & $\mathrm{PhBF}_{3}$ & -3.6 & [21] & -3.6 & 0 & 298.15 \\
\hline Py4-3Me & $\left(\mathrm{CF}_{3} \mathrm{SO}_{2}\right)_{2} \mathrm{~N}$ & -3.7 & [24] & -3.55 & 0.15 & 296.5 \\
\hline Py4-4Me & $\left(\mathrm{CF}_{3} \mathrm{SO}_{2}\right)_{2} \mathrm{~N}$ & -3.69 & [26] & -3.55 & 0.14 & 298 \\
\hline Pip14 & $\left(\mathrm{CF}_{3} \mathrm{SO}_{2}\right)_{2} \mathrm{~N}$ & -3.78 & [22] & -3.51 & 0.27 & 298 \\
\hline IM14 & $\left(\mathrm{CF}_{3} \mathrm{SO}_{2}\right)_{2} \mathrm{~N}$ & -3.54 & [25] & -3.5 & 0.04 & 288.15 \\
\hline IM14 & $\left(\mathrm{CF}_{3} \mathrm{SO}_{2}\right)_{2} \mathrm{~N}$ & -3.5 & & -3.5 & 0 & 293.15 \\
\hline IM14 & $\left(\mathrm{CF}_{3} \mathrm{SO}_{2}\right)_{2} \mathrm{~N}$ & -3.53 & {$[25]$} & -3.5 & 0.03 & 293.15 \\
\hline IM14 & $\left(\mathrm{CF}_{3} \mathrm{SO}_{2}\right)_{2} \mathrm{~N}$ & -3.46 & [27] & -3.5 & -0.04 & 293.15 \\
\hline IM14 & $\left(\mathrm{CF}_{3} \mathrm{SO}_{2}\right)_{2} \mathrm{~N}$ & -3.49 & [29] & -3.5 & -0.01 & 294.15 \\
\hline IM14 & $\left(\mathrm{CF}_{3} \mathrm{SO}_{2}\right)_{2} \mathrm{~N}$ & -3.5 & {$[24]$} & -3.5 & 0 & 296.5 \\
\hline IM14 & $\left(\mathrm{CF}_{3} \mathrm{SO}_{2}\right)_{2} \mathrm{~N}$ & -3.51 & [26] & -3.5 & 0.01 & 298 \\
\hline IM14 & $\left(\mathrm{CF}_{3} \mathrm{SO}_{2}\right)_{2} \mathrm{~N}$ & -3.51 & [25] & -3.5 & 0.01 & 298.15 \\
\hline Pyr14 & $\left(\mathrm{CF}_{3} \mathrm{SO}_{2}\right)_{2} \mathrm{~N}$ & -3.57 & & -3.41 & 0.16 & 293.15 \\
\hline Pyr14 & $\left(\mathrm{CF}_{3} \mathrm{SO}_{2}\right)_{2} \mathrm{~N}$ & -3.59 & {$[22]$} & -3.41 & 0.18 & 298 \\
\hline IM16 & $\mathrm{PF}_{6}$ & -3.45 & [28] & -3.37 & 0.08 & 288.15 \\
\hline IM16 & $\mathrm{PF}_{6}$ & -3.35 & & -3.37 & -0.02 & 293.15 \\
\hline IM16 & $\mathrm{PF}_{6}$ & -3.41 & [28] & -3.37 & 0.04 & 293.15 \\
\hline IM16 & $\mathrm{PF}_{6}$ & -3.36 & [28] & -3.37 & -0.01 & 298.15 \\
\hline IM13 & $\left(\mathrm{CF}_{3} \mathrm{SO}_{2}\right)_{2} \mathrm{~N}$ & -3.29 & [25] & -3.28 & 0.01 & 288.15 \\
\hline IM13 & $\left(\mathrm{CF}_{3} \mathrm{SO}_{2}\right)_{2} \mathrm{~N}$ & -3.28 & [25] & -3.28 & 0 & 293.15 \\
\hline IM13 & $\left(\mathrm{CF}_{3} \mathrm{SO}_{2}\right)_{2} \mathrm{~N}$ & -3.27 & {$[25]$} & -3.28 & -0.01 & 298.15 \\
\hline Py4-4Me & $\left(\mathrm{C}_{4} \mathrm{~F}_{9}\right) \mathrm{SO}_{3}$ & -3.03 & [26] & -3.25 & -0.22 & 298 \\
\hline IM14 & $\left(\mathrm{C}_{4} \mathrm{~F}_{9}\right) \mathrm{SO}_{3}$ & -3.15 & [26] & -3.2 & -0.05 & 298 \\
\hline
\end{tabular}


Table 5. Cont.

\begin{tabular}{|c|c|c|c|c|c|c|}
\hline Cation $^{[a]}$ & Anion $[\mathrm{b}]$ & $\log _{10} x$ & $\begin{array}{l}\text { sat,exp } \\
\text { IL,w }\end{array}$ & $\log _{10} x_{\mathrm{IL}, \mathrm{w}}^{\mathrm{sat} \text { est }}$ & $\Delta_{\text {est,exp }}$ & Temperature $[\mathrm{K}]$ \\
\hline IM12 & $\left(\mathrm{CF}_{3} \mathrm{SO}_{2}\right)_{2} \mathrm{~N}$ & -3.12 & [25] & -3.1 & 0.02 & 288.15 \\
\hline IM12 & $\left(\mathrm{CF}_{3} \mathrm{SO}_{2}\right)_{2} \mathrm{~N}$ & -3.08 & [27] & -3.1 & -0.02 & 293.15 \\
\hline IM12 & $\left(\mathrm{CF}_{3} \mathrm{SO}_{2}\right)_{2} \mathrm{~N}$ & -3.1 & [25] & -3.1 & 0 & 293.15 \\
\hline IM12 & $\left(\mathrm{CF}_{3} \mathrm{SO}_{2}\right)_{2} \mathrm{~N}$ & -3.1 & [24] & -3.1 & 0 & 296.5 \\
\hline IM12 & $\left(\mathrm{CF}_{3} \mathrm{SO}_{2}\right)_{2} \mathrm{~N}$ & -3.08 & [25] & -3.1 & -0.02 & 298.15 \\
\hline Py8-4Me & $\mathrm{CF}_{3} \mathrm{SO}_{3}$ & -3.09 & [21] & -3.09 & 0 & 298.15 \\
\hline Mor11O2 & $\left(\mathrm{CF}_{3} \mathrm{SO}_{2}\right)_{2} \mathrm{~N}$ & -3.19 & & -3.06 & 0.13 & 293.15 \\
\hline Py8-4Me & $\mathrm{BF}_{4}$ & -2.98 & [21] & -3.01 & -0.03 & 298.15 \\
\hline IM18 & $\mathrm{BF}_{4}$ & -2.93 & [30] & -2.9 & 0.03 & 295 \\
\hline IM14 & $\mathrm{PF}_{6}$ & -3 & [28] & -2.87 & 0.13 & 288.15 \\
\hline IM14 & $\mathrm{PF}_{6}$ & -2.8 & [27] & -2.87 & -0.07 & 293.15 \\
\hline IM14 & $\mathrm{PF}_{6}$ & -2.9 & & -2.87 & 0.03 & 293.15 \\
\hline IM14 & $\mathrm{PF}_{6}$ & -2.96 & {$[28]$} & -2.87 & 0.09 & 293.15 \\
\hline IM14 & $\mathrm{PF}_{6}$ & -2.93 & [31] & -2.87 & 0.06 & 294 \\
\hline IM14 & $\mathrm{PF}_{6}$ & -2.87 & [29] & -2.87 & 0 & 294.15 \\
\hline IM14 & $\mathrm{PF}_{6}$ & -2.89 & {$[30]$} & -2.87 & 0.02 & 295 \\
\hline IM14 & $\mathrm{PF}_{6}$ & -2.9 & {$[24]$} & -2.87 & 0.03 & 296.5 \\
\hline IM14 & $\mathrm{PF}_{6}$ & -2.92 & [28] & -2.87 & 0.05 & 298.15 \\
\hline Ру3OH & $\left(\mathrm{CF}_{3} \mathrm{SO}_{2}\right)_{2} \mathrm{~N}$ & -2.43 & & -2.83 & -0.4 & 293.15 \\
\hline $\mathrm{IM} 12 \mathrm{OH}$ & $\left(\mathrm{CF}_{3} \mathrm{SO}_{2}\right)_{2} \mathrm{~N}$ & -2.34 & [24] & -2.66 & -0.32 & 296.5 \\
\hline IM12 & $\mathrm{B}(\mathrm{CN})_{4}$ & -2.46 & [24] & -2.46 & 0 & 296.5 \\
\hline N4444 & $(6-2 \mathrm{Et}) 2 \mathrm{SS}$ & -1.52 & {$[32]$} & -1.52 & 0 & 298 \\
\hline
\end{tabular}

[a] Cation acronyms explained in Table 1

[b] (6-2Et)-2SS = bis(2-ethylhexyl)sulfosuccinate

\section{References and Notes}

1. Walden, P. Ueber die Molekulargrösse und elektrische Leitfähigkeit einiger geschmolzener Salze (Molecular weights and electrical conductivity of several fused salts). Bull Acad. Imper. Sci. St. Petersburg 1914, 8, 405-422.

2. Wilkes, J.S.; Zaworotko, M.J. Air and water stable 1-Ethyl-3-Methylimidazolium based ionic liquids. J. Chem. Soc. Chem. Comm. 1992, 1992, 965-967.

3. Heintz, A. Recent developments in thermodynamics and thermophysics of non-aqueous mixtures containing ionic liquids. A review. J. Chem. Thermodyn. 2005, 37, 525-535.

4. Ludwig, R.; Kragl, U. Do we understand the volatility of ionic liquids? Angew. Chem. Int. Ed. 2007, 46, 6582-6584. 
5. Ludwig, R.; Kragl, U. Verstehen wir die Flüchtigkeit ionischer Flüssigkeiten? Angew. Chem. 2007, 119, 6702-6704.

6. Rebelo, L.P.; Lopes, J.N.; Esperanca, J.M.; Guedes, H.J.; Lachwa, J.; Najdanovic-Visak, V.; Visak, Z.P. Accounting for the unique, doubly dual nature of ionic liquids from a molecular thermodynamic and modeling standpoint. Acc. Chem. Res. 2007, 40, 1114-1121.

7. Padua, A.A.; Costa Gomes, M.F.; Canongia-Lopes, J.N. Molecular solutes in ionic liquids: a structural perspective. Acc. Chem. Res. 2007, 40, 1087-1096.

8. Weingärtner, H. Understanding ionic liquids at the molecular level: Facts, problems, and controversies. Angew. Chem. Int. Ed. 2008, 47, 654-670.

9. Deetlefs, M.; Seddon, K.R.; Shara, M. Predicting physical properties of ionic liquids. Phys. Chem. Chem. Phys. 2006, 8, 642-649.

10. Slattery, J.M.; Daguenet, C.; Dyson, P.J.; Schubert, T.J.; Krossing, I. How to predict the physical properties of ionic liquids: a volume-based approach. Angew. Chem. Int. Ed. Engl. 2007, 46, 5384-5388.

11. Lynden-Bell, R.M.; Del Popolo, M.G.; Youngs, T.G.; Kohanoff, J.; Hanke, C.G.; Harper, J.B.; Pinilla, C.C. Simulations of ionic liquids, solutions, and surfaces. Acc. Chem. Res. 2007, 40, 11381145 .

12. Bruzzone, S.; Malvaldi, M.; Chiappe, C. A RISM approach to the liquid structure and solvation properties of ionic liquids. Phys. Chem. Chem. Phys. 2007, 9, 5576-5581.

13. Couling, D.J.; Bernot, R.J.; Docherty, K.M.; Dixon, J.K.; Maginn, E.J. Assessing the factors responsible for ionic liquid toxicity to aquatic organisms via quantitative structure - property relationship modeling. Green Chem. 2006, 8, 82-90.

14. Ranke, J.; Stock, F.; Müller, A.; Stolte, S.; Störmann, R.; Bottin-Weber, U.; Jastorff, B. Lipophilicity parameters for ionic liquid cations and their correlation to in vitro cytotoxicity. Ecotoxicol. Environ. Safety 2007, 67, 430-438.

15. García-Lorenzo, A.; Tojo, E.; Tojo, J.; Teijeira, M.; Rodríguez-Berrocal, F.J.; Pérez González, M.; Martínez-Zorzano, V.S. Cytotoxicity of selected imidazolium-derived ionic liquids in the human Caco-2 cell line. Sub-structural toxicological interpretation through a QSAR study. Green Chem. 2008, 10, 508-516.

16. Ranke, J.; Stolte, S.; Stormann, R.; Arning, J.; Jastorff, B. Design of sustainable chemical products — the example of ionic liquids. Chem. Rev. 2007, 107, 2183-2206.

17. Harjani, J.R.; Singer, R.D.; Garcia, M.T.; Scammells, P.J. The design and synthesis of biodegradable pyridinium ionic liquids. Green Chem. 2008, 10, 436-438.

18. Kalidas, C.; Hefter, G.; Marcus, Y. Gibbs energies of transfer of cations from water to mixed aqueous organic solvents. Chem. Rev. 2000, 100, 819-852.

19. Stolte, S.; Arning, J.; Bottin-Weber, U.; Pitner, W.; Welz-Biermann, U.; Jastorff, B.; Ranke, J. Effects of different head groups and modified side chains on the cytotoxicity of ionic liquids. Green Chem. 2007, 9, 760-767.

20. Branco, L.C.; Rosa, J.N.; Ramos, J.J.M.; Afonso, C.A.M. Preparation and characterization of new room temperature ionic liquids. Chem. Eur. J. 2002, 8, 3671-3677. 
21. Papaiconomou, N.; Salminen, J.; Lee, J.-M.; Prausnitz, J.M. Physicochemical properties of hydrophobic ionic liquids containing 1-octylpyridinium, 1-octyl-2-methylpyridinium, or 1-octyl-4methylpyridinium cations. J. Chem. Eng. Data 2007, 52, 833-840.

22. Salminen, J.; Papaiconomou, N.; Kumara, R.A.; Lee, J.; Kerr, J.; Newmana, J.; Prausnitz, J.M. Physicochemical properties and toxicities of hydrophobic piperidinium and pyrrolidinium ionic liquids. Fluid Phase Equilib. 2007, 261, 421-426.

23. Kakiuchi, T.; Tsujioka, N.; Kurita, S.; Iwami, Y. Phase-boundary potential across the nonpolarized interface between the room-temperature molten salt and water. Electrochem. Commun. 2003, $5,159-164$.

24. Chapeaux, A.; Simoni, L.D.; Stadtherr, M.A.; Brennecke, J.F. Liquid phase behavior of ionic liquids with water and 1-Octanol and modeling of 1-Octanol/Water partition coefficients. J. Chem. Eng. Data 2007, 52, 2462-2467.

25. Freire, M.G.; Carvalho, P.J.; Gardas, R.L.; Marrucho, I.M.; Santos, L.M.N.B.F.; Coutinho, J.A.P. Mutual solubilities of water and the $[\mathrm{c}(\mathrm{n}) \mathrm{mim}][\mathrm{tf}(2) \mathrm{n}]$ hydrophobic ionic liquids. J. Phys. Chem. B 2008, 112, 1604-1610.

26. Papaiconomou, N.; Yakelis, N.; Salminen, J.; Bergman, R.; Prausnitz, J.M. Synthesis and properties of seven ionic liquids containing 1-methyl-3-octylimidazolium or 1-butyl-4-methylpyridinium cations. J. Chem. Eng. Data 2006, 51, 1389-1393.

27. Luo, H.; Dai, S.; Bonnesen, P.V. Solvent extraction of Sr2+ and Cs+ based on room-temperature ionic liquids containing monoaza-substituted crown ethers. Anal. Chem. 2004, 76, 2773-2779.

28. Freire, M.G.; Neves, C. M. S.S.; Carvalho, P.J.; Gardas, R.L.; Fernandes, A.M.; Marrucho, I.M.; Santos, L. M. N. B.F.; Coutinho, J. A.P. Mutual solubilities of water and hydrophobic ionic liquids. J. Phys. Chem. B 2007, 111, 13082-13089.

29. Shvedene, N.V.; Borovskaya, S.V.; Sviridov, V.V.; Ismailova, E.R.; Pletnev, I.V. Measuring the solubilities of ionic liquids in water using ion-selective electrodes. Anal. Bioanal. Chem. 2005, $381,427-430$.

30. Anthony, J.L.; Maginn, E.J.; Brennecke, J.F. Solution thermodynamics of imidazolium-based ionic liquids and water. J. Phys. Chem. B 2001, 105, 10942-10949.

31. Carda-Broch, S.; Berthod, A.; Armstrong, D.W. Solvent properties of the 1-butyl-3-methylimidazolium hexafluorophosphate ionic liquid. Anal. Bioanal. Chem. 2003, 375, 191-199.

32. Nishi, N.; Kawakami, T.; Shigematsu, F.; Yamamoto, M.; Kakiuchi, T. Fluorine-free and hydrophobic room-temperature ionic liquids, tetraalkylammonium bis(2-ethylhexyl)sulfosuccinates, and their ionic liquid-water two-phase properties. Green Chem. 2006, 8, 349-355.

33. Kakiuchi, T. Ionic-liquid vertical bar water two-phase systems. Anal. Chem. 2007, 79, 6442-6449.

(c) 2009 by the authors; licensee Molecular Diversity Preservation International, Basel, Switzerland. This article is an open-access article distributed under the terms and conditions of the Creative Commons Attribution license (http://creativecommons.org/licenses/by/3.0/). 